\title{
Cavernous Angioma of the Cerebellum and Cerebellar Atrophy
}

\author{
-Case Report-
}

\author{
Akihiro TAKAHASHI, Hiroyasu KamiYAMA, Hiroshi ABE, \\ Satoshi KURODA*, Hiroyuki IMAMURA* and Hisatoshi SAITOH* \\ Department of Neurosurgery, Hokkaido University School of Medicine, Sapporo; \\ ${ }^{*}$ Sapporo Azabu Neurosurgical Hospital, Sapporo
}

\begin{abstract}
A cavernous angioma of the cerebellum occurred in a 55-year-old female presenting with a 3-year history of slowly progressive cerebellar signs and symptoms. Computed tomography and magnetic resonance imaging revealed a lesion in the left cerebellar nuclei and atrophic change of the affected cerebellar hemisphere. The final diagnosis was made at operation. The cerebellar nuclei and white matter were probably affected by the slowly expanding lesion, with repeated hemorrhage leading to degeneration of the afferent and efferent fibers, and subsequent atrophy of the affected cerebellar hemisphere.
\end{abstract}

Key words: cavernous angioma, cerebellar atrophy, silent hemorrhage

\section{Introduction}

Cerebellar cavernous angioma is relatively rare, with only 10 histopathologically confirmed cases reported. ${ }^{3)}$ We describe a unique case of cerebellar cavernous angioma presenting with a 3-year history of slowly progressive cerebellar signs and symptoms contributory to cerebellar hemispheric atrophy.

\section{Case Report}

A 55-year-old female complained of abnormal speech and movement difficulties of her left hand. She never had headaches, nausea, or dizziness. Neurological examination disclosed scanning speech and left lower extremity ataxia. A precontrast computed tomographic (CT) scan showed a hyperdense lesion in the left cerebellar nuclei and cerebellar atrophy. The lesion was slightly enhanced on postcontrast CT scan (Fig. 1). $T_{2}$-weighted magnetic resonance (MR) images revealed hyperintensity surrounded by extensive hypointensity and atrophic change (Fig. 2). Vertebral angiograms showed no significant abnormalities.

Received January 29, 1992

Accepted April 6, 1992
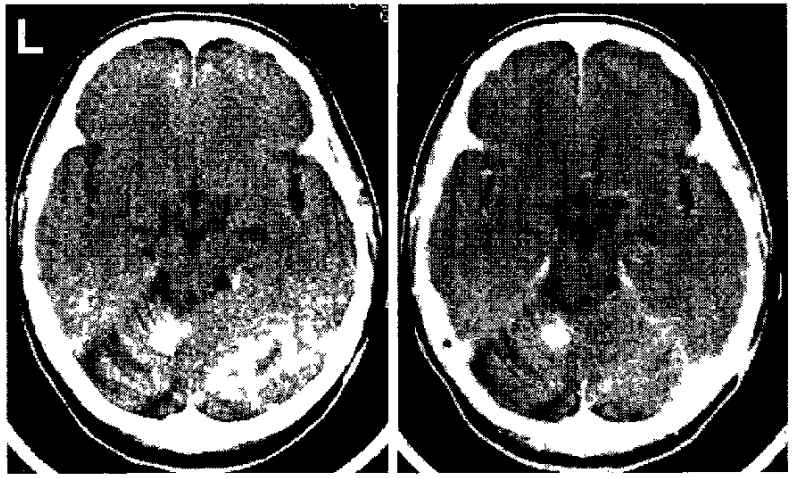

Fig. I left: Precontrast axial CT scan, showing a slightly dense lesion in the left cerebellar nuclei and atrophy of the affected cerebellar hemisphere. right: Postcontrast axial CT scan, demonstrating slight enhancement.

The lesion was removed through a left occipital transtentorial approach with the patient in the prone position. Histological examination showed evidence of cavernous angioma. A number of blood vessels with thickened tunicae intimae and organized thrombi were detected. Hemosiderin was deposited in the parenchyma of the lesion, mainly around the vessels 


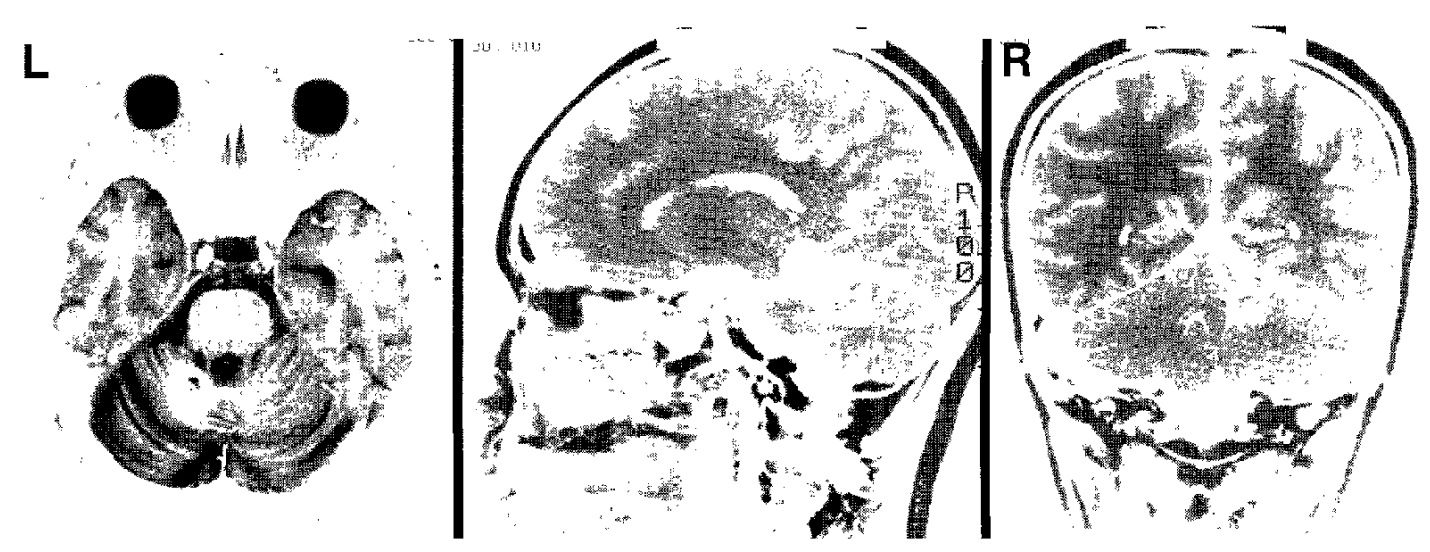

Fig. 2 left: Axial $\mathrm{T}_{2}$-weighted MR image (SE $3000 / 80 \mathrm{msec}$ ), demonstrating hyperintense center surrounded by extensive peripheral hypointensity, and atrophy of the left cerebellar hemisphere. center, right: Sagittal (center) and coronal (right) $\mathrm{T}_{1}$-weighted MR images (SE 500/20 $\mathrm{msec}$ ), demonstrating isointense central foci with surrounding hypointensity.

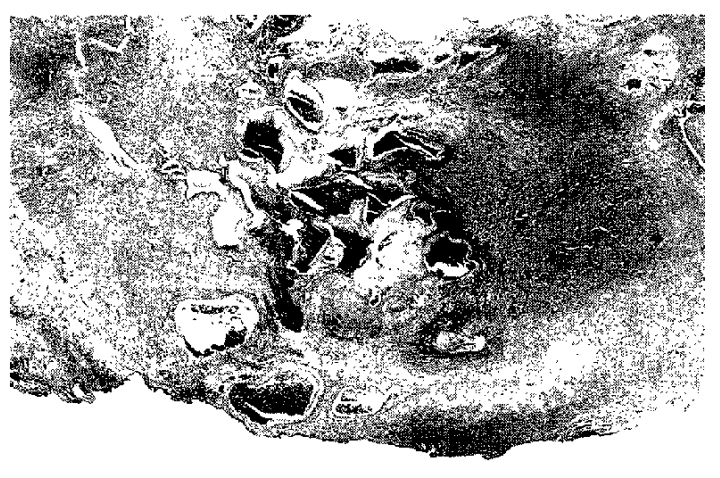

Fig. 3 Photomicrograph of the surgical specimen, showing a number of blood vessels with thickened tunicae intimae. Calcification is not observed, but organized thrombi and hemosiderin deposits are visible. van Gieson stain, $\times 27$.

(Fig. 3).

She had an uneventful postoperative course and was discharged without neurological changes 1 month later.

\section{Discussion}

Cavernous angioma of the cerebellum is relatively rare. Sakai et al. ${ }^{8)}$ reported that the location of 248 intracranial cavernous angiomas was: $80 \%$ above the tentorium, $10 \%$ in the brainstem, $4 \%$ in the cerebellum, and $6 \%$ in multiple regions.

The presenting signs and symptoms vary according to the anatomical location. In some cases, the initial onset is sudden, while others are progressive. ${ }^{5)}$ Giombi and Morello $^{2)}$ reported that the initial symptoms of cavernous angioma were: seizures, $38 \%$; headache, $28 \%$; hemorrhage, $24 \%$; and local signs, $12 \%$. There are few clinical reports of cerebellar cavernous angioma. Ishikawa et al. ${ }^{3)}$ reviewed nine reported cases with adequate clinical and histopathological descriptions and one case of their own. Onset was sudden in all 10 patients, and in most cases headache was the initial symptom, often accompanied by dizziness or vomiting.

Clinical reports of intracranial cavernous angioma associated with brain atrophy are limited. Bicknell et $a l{ }^{11}$ described a case of cavernous angioma located in the head of the right caudate nucleus associated with ventricular enlargement, but this was possibly due to a long history of alcohol abuse with no significant causal relationship to the angioma. Vanquero et al. ${ }^{10)}$ analyzed CT scans of 24 patients with surgically treated intracranial cavernous angiomas, and found perilesional hypodensities suggestive of brain tissue atrophy in $22 \%$. However, there are no reported cases associated with ipsilateral cerebral or cerebellar hemiatrophy.

Cerebellar atrophy has a variety of etiologies. ${ }^{7)}$ Olivopontocerebellar atrophy usually occurs later in life and may be either familial or sporadic. Atrophy of the pons and inferior olivary nuclei together with cerebellar atrophy involve mainly the anterior and posterior lobes. Parenchymatous cerebellar atrophy may be associated with toxins such as alcohol or the use of phenytoin, with the major pathology in alcoholic degeneration located in the superior vermis. Vascular lesions and postoperative defects may 
result in focal atrophy. Some germinomas in the thalamus or basal ganglia are associated with ipsilateral cerebral atrophy. Kwak et al. ${ }^{4)}$ suggested that slowly growing tumors caused degeneration and disappearance of ganglion cells and nerve fibers, and secondary Wallerian degeneration of afferent and projecting fibers occurred, resulting in extensive atrophy of the cerebral cortex and subcortical tissue.

The cavernous angioma in this case originated in the cerebellar nuclei and caused a 3-year history of slowly progressive signs and symptoms. Deposition of hemosiderin around the lesion suggested that repeated clinically silent hemorrhages ${ }^{6,9)}$ had occurred. The cerebellar nuclei and white matter were probably affected by the slowly expanding lesion, with repeated hemorrhage leading to degeneration of the afferent and efferent fibers, and subsequent atrophy of the affected cerebellar hemisphere.

\section{References}

1) Bicknell JM, Carlow TJ, Kornfeld M, Stovring J, Turner P: Familial cavernous angiomas. Arch Neurol 35: 746-749, 1978

2) Giombi S, Morello G: Cavernous angioma of the brain. Account of fourteen personal cases and review of the literature. Acta Neurochir (Wien) 40: 61-82, 1978

3) Ishikawa S, Kuwabara S, Fukuma A, Seo H, Andoh $\mathrm{S}$ : Cavernous angioma of the cerebellum. Case report. Neurol Med Chir (Tokyo) 29: 35-39, 1989
4) Kwak R, Saso S, Suzuki J: Ipsilateral cerebral atrophy with thalamic tumor of childhood. $J$ Neurosurg 48: 443-449, 1978

5) Lobato R, Perez C, Rivas JJ, Cordobes F: Clinical, radiological and pathological spectrum of angiographically occult intracranial vascular malformations. $J$ Neurosurg 68: 518-531, 1988

6) Rigamonti D, Drayer BP, Johnson PC, Hadley MN, Zabramski J, Spetzler RF: The MRI appearance of cavernous malformations (angiomas). $J$ Neurosurg 67: 518-524, 1987

7) Rothman SLG, Glanz S: Cerebellar atrophy: The differential diagnosis by computerized tomography. Neuroradiology 16: 123-126, 1987

8) Sakai N, Asano Y, Tanigawara $T$, Andoh T, Yamada H: Clinical study of cavernous angioma. No Socchu 13: 52-57, 1991 (in Japanese)

9) Steiger HJ, Markwalder TM, Reulen HJ: Clinicopathological relations of cerebral cavernous angiomas: Observations in eleven cases. Neurosurgery 21: 879-883, 1987

10) Vanquero J, Salazar J, Martinez R, Martinez P, Bravo G: Cavernoma of the central nervous system: Clinical syndromes, CT scan diagnosis, and prognosis after surgical treatment in 25 cases. Acta Neurochir (Wien) 85: 29-33, 1987

Address reprint requests to: A. Takahashi, M.D., Department of Neurosurgery, Hokkaido University School of Medicine, North-15, West-7, Kita-ku, Sapporo 060 , Japan. 